MARISA FAGGINI

Working paper No. 25 - October 2011 


\title{
Chaotic Time Series Analysis in Economics: Balance and Perspectives
}

\author{
Marisa Faggini \\ Dipartimento di Scienze Economiche e Statistiche \\ Università di Salerno \\ email: mfaggini@unisa.it
}

\begin{abstract}
To show that a mathematical model exhibits chaotic behaviour does not prove that chaos is also present in the corresponding data. To convincingly show that a system behaves chaotically, chaos has to be identified directly from the data. From an empirical point of view, it is difficult to distinguish between fluctuations provoked by random shocks and endogenous fluctuations determined by the nonlinear nature of the relation between economic aggregates. For this purpose, chaos tests test are developed to investigate the basic features of chaotic phenomena: nonlinearity, fractal attractor, and sensitivity to initial conditions.

The aim of the paper is not to review the large body of work concerning nonlinear time series analysis in economics, about which much has been written, but rather to focus on the new techniques developed to detect chaotic behaviours in the data. More specifically, our attention will be devoted to reviewing the results reached by the application of these techniques to economic and financial time series and to understand why chaos theory, after a period of growing interest, appears now not to be such an interesting and promising research area.
\end{abstract}

Key words: economic dynamics, nonlinearity, tests for chaos, chaos

JEL code: B59, E20

\section{Introduction}

The approaches used to address the analysis of economic time series can be classified into two main categories: linear and nonlinear. Linear methods allow one to grasp all the regular structures in a data set assuming that the intrinsic dynamics of a system are governed by linear laws and, most importantly, that small causes produce small effects ${ }^{1}$. However, these methods that investigate the dynamics of time series often leave significant features unexamined and unexploited, highlighting the fact that real economic time series do not show the kind of regularity assumed by them. Irregular frequencies and different amplitudes are the real features of fluctuations in economic data.

To explain the stochasticity in real-life observations, economists have included stochastic considerations in their speculations. However, these stochastic considerations that have

\footnotetext{
Kantz H., Schreiber T., (1997)
} 
entered economic analysis are very much a compromise. Stochastic exogenous disturbances were superimposed upon usual linear deterministic models to produce the stochastic appearance of real economic time series. However, the linear stochastic equations were shown to be inadequate to fit the economic phenomena well. Therefore, alternative answers were sought by economists in the nonlinear approach.

In the literature, there many examples of nonlinear methods used to analyse time series. These are the so-called "ARCH-type" models (autoregressive conditional heteroskedasticity models) proposed by Engle (1982) and generalised by Bollerslev (1986). Among the "ARCH-type" models - Exponential GARCH, Asymmetric Power ARCH, Threshold GARCH - the so-called integrated model (IGARCH) and fractionally integrated model (FIGARCH) have been the most popular recently. These models are based on the assumption that data are nonlinear stochastic functions of their past values.

However, it is also possible that data can be generated by deterministic processes. Nonlinear deterministic systems with a few degrees of freedom can create output signals that appear complex and mimic stochastic signals from the point of view of conventional time series analysis. Certain deterministic nonlinear systems may show chaotic behaviours, or better qualitative different behaviours are produced by one or more parameters. There are different signs, or invariants, that are representative of chaos in a system: nonlinearity, dependence on initial conditions, and presence of an attractor with fractal dimension, known as a strange attractor. Based on these signs, several tools have been developed in recent years to investigate the chaotic properties of a system from a time series. These tools are generally divided into two categories: metric and topological. The first, which includes the Lyapunov exponent, the correlation dimension, and BDS tests, is characterised by studying the distances between points on the attractor. The second, which is similar to the close return test and recurrence analysis, though based on the geometric properties of the asymptotic structure of a dynamic system, is characterised also by being applicable to short data sets.

Consequently, it is fair to assume that a time series with a seemingly random pattern, an inherent nonlinear dependence, lower estimates for correlation dimension, and positive estimates of the largest Lyapunov exponent could be a manifestation of underlying chaotic dynamics.

Because as researchers we are interested in gaining better insight and understanding of the underlying dynamics of economic phenomena to provide reliable predictions, we must be conscious of different approaches and tools to perform this goal. Thus, our aim is not to review the large body of works concerning nonlinear time series analysis, about which much has been written, but rather to focus on those analysis techniques developed to detect chaotic behaviours in economic data. More specifically, our attention will be devoted to reviewing the basic chaos tests, highlighting their ability and limitations, and studying the results reached by their application in analysing economic and financial time series. The motivation is to understand why chaos theory, after a period of growing interest, appears now to be not such an interesting and promising research area in economics.

The rest of this paper is organised as follows. In Section 2, the main methods used in chaotic tests are introduced and evaluated. Sections 3 and 4 synthesise the results of application of 
chaotic tests in economic and financial data. In Section 5, the pitfalls in the application of these methods are highlighted, and some concluding comments are presented in Section 6.

\section{New tools for analysing economic time series}

Although it sounds like an oxymoron, the concept of deterministic chaos has gained popularity in many fields, including economics and finance. This is because chaos theory stimulates the search for a mechanism that generates observed movements in real economic data and that minimises the role of exogenous shocks. In this sense, it represents a shift in thinking about methods for studying economic activity and in the explanation of economic phenomena such as fluctuations, instability, crises, and depressions.

The behaviour of chaotic systems does not unfold in a linearly predictable, conventional cause-and-effect manner over time. When viewed as a whole, these systems manifest definite patterns and structures, but their future direction cannot be predicted from their past history.

While the stochastic trends of irregular systems are explained by random shocks external to the systems, in chaotic systems, the fluctuations are within the system. They are the result of complex interactions among the system's elements, and although it is difficult to predict system behaviour, the same cannot be said of the process that created it, as it is deterministic. There is no standard definition of chaos (Ditto and Munakata, 1995). It is generally associated with systems with boundaries (Cheng and Tong, 1992) that are ergodic (Jensen, 1993), characterised by a strange attractor with dynamics sensitive to initial conditions (Eckmann and Ruelle, 1985) and a positive Lyapunov exponent (Wolf et al., 1985).

In 1986, Devaney defined $f: X \rightarrow X$ as a chaotic map in $\mathrm{X}$ if

- $\quad$ (i) $f$ is characterised by sensitivity to initial conditions;

- $\quad$ (ii) $f$ is topologically transitive;

- $\quad$ (iii) the set of points of $f$ are dense in $X$.

In the Devaney definition, some basic features of chaos systems are stressed, such as unpredictability (i), indecomposability (ii), and deterministic behaviour (iii). A more complete definition is provided by Kellert (1992), who defines chaos theory as the qualitative study of unstable aperiodic behaviour in deterministic nonlinear dynamical systems.

Therefore, we can consider a system to be chaotic

- If it is nonlinear. Of course, not all nonlinear systems are characterised by chaos, but it is certain that a linear system cannot be chaotic.

- If is generated by deterministic rules. If we know the state of the universe at some initial time, we should be able to determine its state at any other time.

- If is sensitive to initial conditions. To be deterministic in the case of chaotic systems does not mean to be predictable, but small changes in the initial state can lead to radically different behaviour in the final state. This sensitivity ${ }^{2}$ to initial conditions

\footnotetext{
${ }^{2}$ "Recently a class of systems have been discovered whose behaviour is much more complex and fails the continuity condition in a radical way. These are the chaotic systems. No matter how close two systems are
} 
makes such systems unpredictable and therefore difficult to follow their evolution in the time.

- If is characterised by a strange attractor. To say that a system is unpredictable is not to say that it lacks coherence or structure. Nonlinear systems that appear to explode into unpredictable outcomes do possess a deep structure, called an attractor. An attractor is an organising principle, an inherent shape or state to which a phenomenon will always tend to return as it evolves. In particular, chaotic systems are characterised by strange attractors that are topologically distinct from a periodic orbit or a limit cycle. Such constructs are called fractal attractors because they have a fractal dimension.

Economists have used linear equations to model most economic phenomena, even if they are inherently nonlinear, because these equations are easy to manipulate and usually yield unique solutions. When the irregular behaviour of some nonlinear relations is found, it is not appreciated by economists because it is difficult and intractable to deal with, so it is explained as being stochastic or linearised. Conceptualising, measuring, and modelling cause-effect linear relationships in economic systems is sometimes ineffective and inefficient. On the contrary, it is usually closer to reality to propose that relationships among the economic agents and variables are nonlinear. By the 1980s, physics had offered new suggestions for a more realistic understanding and modelling of economic phenomena. The spread of non-equilibrium thermodynamic ideas and the increasing interest of all scientific fields in nonlinear dynamic models with the discovery that simple nonlinear models can show complex dynamics pushed economists to study nonlinear dynamics and chaos theory as a possible framework for modelling economic phenomena.

Economists began to look at chaotic analyses of the late 1970s and the 1980s, including such important works as those by Medio (1979), Stutzer (1980), Benhabib and Day (1981), Day (1982), and Grandmont (1985), just to name few. A common feature of chaos models is that nonlinear dynamics tend to arise as the result of relaxing the assumptions underlying the competitive market general equilibrium approach.

The relevance of addressing chaos in economic models is associated with detecting the presence of chaotic motion in economic data. To show that a mathematical model exhibits chaotic behaviour does not prove that chaos is also present in the corresponding experimental data. To convincingly show that a system behaves chaotically, chaos has to be identified directly from the data.

From an empirical point of view, it is difficult to distinguish between fluctuations provoked by random shocks and endogenous fluctuations determined by the nonlinear nature of the relation between economic aggregates. For this purpose, chaos tests test are developed to investigate the basic features chaotic phenomena: nonlinearity, fractal attractor, and sensitivity to initial conditions.

initially, their phase space paths may diverge arbitrarily far. [...] Between two points whose path ends later in say, area A there will be a point whose paths ends in B ...and vice versa. This phenomenon will occur no matter how close the chosen points are", cit. in Stone, 1989, p. 128. 


\subsection{Correlation Dimension}

A necessary but not sufficient condition in order to define a system as being chaotic is that the strange attractor has a fractal dimension.

The notion of dimension refers to the degree of complexity of a system expressed by the minimum number of variables that is needed to replicate the system (Schwartz and Yousefi, 2003). For example, a cube has three dimensions, a square has two dimensions, and a line has one. The topological dimension is always an integer. A chaotic system has non-integer dimensionality called fractal dimension. The fractal dimension measures the probability that two points chosen at random will be within a certain distance of each other and examines how this probability changes as the distance is increased.

In the literature, there are many methods ${ }^{3}$ for calculating the fractal dimension (Hausdorff dimension, the box-counting dimension, the information dimension, and the correlation dimension), which nevertheless do not provide equivalent measures (Hentschel - Procaccia, 1983). Among these different algorithms, the correlation dimension proposed by Grassberger-Procaccia (1983), based on phase space reconstructions of the process to estimates $^{4}$, has the advantage of being straightforward and quickly implemented.

Let us consider the mono-dimensional series $\left\{x_{t}\right\}_{t=1}^{n}$ and, from this, the sequence of $N=n-m+1 \quad m$-dimensional vectors, $X_{t}=\left(x_{t}, x_{t-1}, \ldots, x_{t-m+1}\right)$ that gives us the reconstructed series $\left\{x_{t}\right\}_{t=m}^{n}$.

If the unknown system that generated $\left\{x_{t}\right\}_{t=1}^{n}$ is $\mathrm{n}$-dimensional, and provided that embedding dimension $^{5}$ is $m \geq 2 n+1^{6}$, we have that the set of m-histories recreates the dynamics of the data-generating system and can be used to analyse its dynamics (Pachard et al., 1980, Takens, 1981).

Let us suppose that $C(N, m, \varepsilon)$ is the number of points separated by a distance less than $\varepsilon$; for a given embedding dimension, the correlation function ${ }^{7}$ is given by

$$
C(N, m, \varepsilon)=\frac{1}{N(N-1)} \sum_{m \leq t \neq s \leq N} H\left(\varepsilon-\left\|X_{t}-X_{s}\right\|\right) \quad \varepsilon>0
$$

\footnotetext{
3 See Farmer et al., 1983, Cutler, 1991, Barnsley, 1988, and Falconer, 1990.

${ }^{4}$ This procedure is based upon the method of delay time coordinates, by Takens (1981) that showed that this type of reconstruction yields a topological equivalent attractor leaving the dynamic parameters invariant.

${ }^{5}$ Basic elements to reconstruct the time series from the original one are the delay-time and the embedding dimension. In the literature there are some techniques like the False Nearest Neighbor and the Mutual Information Function in order to choose respectively the embedding dimension and the delay-time.

${ }^{6}$ According to the numerical results provided by Packard et al.,(1980) it is possible to get reasonable results with much smaller embedding dimensions. This point is particularly interesting in different economic applications since in such cases the dimension of the true phase space is often not known a priori. Over the years this insight has widely been adopted in economic literature on chaos where common practice is to choose $\mathrm{m}$ around 10-12. (Schwartz and Yousefi 2003).

${ }^{7}$ Example by Barnett and He, 2000.
} 
$H(z)$ is the Heaviside function given by $H(z)=1$ for all $z \geq 0$ and 0 ; otherwise, $\varepsilon$ is the sufficiently small distance between vectors $X_{t}$ and $X_{s}$, and $\|$.$\| is the norm operator.$

The correlation function $C(N, m, \varepsilon)$ gives the probability that a randomly selected pair of delay coordinate points is separated by a distance less than $\varepsilon$. It measures the frequency with which temporal patterns are repeated in the data.

To determine the correlation dimension from (6), we have to determine how $C(N, m, \varepsilon)$ changes as $\varepsilon$ changes. As $\varepsilon$ grows, the value of $C(N, m, \varepsilon)$ grows because the number of near points to be included in (6) increases. Grassberger and Procaccia (1983) show that for sufficiently small $\varepsilon, C(N, m, \varepsilon)$ grows at rate $D_{C}$ and can be well approximated by $C(N, m, \varepsilon) \approx \varepsilon^{D_{C}}$

That is, the correlation function is proportional to the same power of $D_{C}$ that represents the value of the correlation dimension.

More formally, the dimension associated with the reconstructed dynamic is given by

$$
D_{C}=\lim _{\varepsilon \rightarrow 0} \frac{\log C(N, m, \varepsilon)}{\log \varepsilon}
$$

That is, it is given by the slope of the regression of $\log C(N, m, \varepsilon)$ versus $\log \varepsilon$ for small values of $\varepsilon$ and depends on the chosen embedding dimension.

If, as $m$ increases $D_{C}$ continues to rise, then this relationship is symptomatic of a stochastic system. If the data are generated by a chaotic system, $D_{C}$ will reach a finite limit at some relatively small $m$ (saturation point). The importance of the correlation dimension arises from the fact that the minimum number of variables required to model a chaotic attractor is the smallest integer greater than the correlation dimension itself.

The reliability of implementing this algorithm suffers from some problems. Because it is based on the method of delay time coordinates introduced by Takens (1981), the estimates of the embedding dimension and delay time are so crucial that an unfortunate delay time choice yields misleading results concerning the dimension of well-known attractors.

Other than the problems associated with these estimates, the correlation dimension suffers from two other problems related to the choice of sufficiently small $\varepsilon$ and the norm operator. With the limited length of the data, it will almost always be possible to select sufficiently small $\varepsilon$ so that any two points will not lie within $\varepsilon$ of each other (Ramsey and Yuan, 1989).

Regarding the norm operator, while Brock's (1986) theorem gives the conditions under which the correlation function remains independent of the choice of norm, Kugiumtzis (1997) shows the invalid application of this theorem for short noisy time series, such as economic and financial series. Therefore, under such circumstances, the most reliable results are obtained by using the Euclidian norm (Schwartz and Yousefi, 2003). The presence of noise in time series not obtained from experiments could further compromise the distinction between stochastic and deterministic behaviour. Therefore, a data-filtering procedure (linear 
and nonlinear) is required to reduce the unwelcome noise level without distorting the original signal $^{8}$ to obtain reliable results.

Reliability could also be compromised by using short data sets (Ramsey and Yuan 1989, 1990). In fact, in the case of high-dimensional chaos, it will be very difficult to make estimates without an enormous amount of data. This suggests that the correlation dimension can only distinguish low-dimensional chaos from high-dimensional stochastic processes, particularly with economic data. Furthermore, if the fractal dimension is found, the correlation dimension, as in all nonparametric methods, does not provide information about the dynamics of the process that generated it because it does not preserve time-ordering data (Gilmore 1993a, b).

\subsection{BDS Test}

The BDS test ${ }^{9}$ introduced by Brock, Dechert and Scheinkman $(1987,1996)$ is a nonparametric method based on the correlation function developed by Grassberger-Procaccia (1983), defined in (6), and used to test for serial dependence and nonlinear structures ${ }^{10}$ in a time series. The BDS test incorporates the embedding dimensions, but it assumes the delay time equals $1^{11}$.

Therefore, the BDS test is not considered to be a direct test for chaos; rather, it is used as a model selection tool to obtain some information about what kind of dependency exists after removing nonlinear dependency from the data.

The standardised residuals from an ARCH-type model are extracted and then tested for nonlinear dependence. If there is no dependence, the data are not chaotic because the ARCHtype model has captured all nonlinearities (Hsieh, 1989, 1991); otherwise, the BDS test is applied to residuals to check if the best-fit model for a given time series is a linear or nonlinear model.

The BDS tests the null hypothesis that the variable of interest is independently and identically distributed (IID). Because IID implies randomness, if a series is proved to be IID, it is random (Barnett et al., 1997).

Under the null hypothesis of whiteness, the BDS statistic is obtained by ${ }^{12}$

$$
W(N, m, \varepsilon)=\sqrt{N} \frac{C(N, m, \varepsilon)-C(N, 1, \varepsilon)^{m}}{\hat{\sigma}(N, m, \varepsilon)}
$$

\footnotetext{
${ }^{8}$ Caputo et al. forthcoming in Chaos Solitons and Fractals

9 Subsequent to its introduction, the BBS test was generalised by Savit and Green (1991) and Wu, Savit, and Brock (1993) and more recently, DeLima (1998) introduced an iterative version of the BBS test.

${ }^{10}$ "There are three particularly well known tests currently in use for testing for nonlinearity: BDS test, White's neural network test and the Hinich bispectrum tes, Barnett et al., 1997, p. 8.

${ }^{11}$ See Barnett et al. (1997) and Matilla-Garcia et al. (2004) for the problems when fixing delay time to one. Moreover we have to consider The BDS-G test suggested by Matilla-Garcià et al. (2004) as a new way for selecting an adequate delay time which allows to obtain a good approximation of the correlation dimension.

${ }^{12}$ Example by Barnett and $\mathrm{He}, 2000$
} 
The correlation function asymptotically follows standard normal distribution $N(0,1)$ : $\lim _{N \rightarrow \infty} W(N, m, \varepsilon) \sim N(0,1), \forall m, \varepsilon . \hat{\sigma}(N, m, \varepsilon)^{13}$ is the standard sample deviation of $C(N, m, \varepsilon)-C(N, 1, \varepsilon)^{m}$

Moving from the hypothesis that a time series is IID, the BDS tests the null hypothesis that $C(N, m, \varepsilon)=C(N, 1, \varepsilon)^{m}$, which is equivalent to the null hypothesis of whiteness against an unspecified alternative.

Hsieh (1991) shows that the BDS test can detect the presence of four types of non-IID behaviours resulting from a non-stationarity of the series, a linear stochastic system (such as ARMA processes), a nonlinear stochastic system (such as ARCH/GARCH processes), or a nonlinear deterministic system, which could feature low-order chaos ${ }^{14}$. If series are IID. in which linear or even conditional heteroskedasticity can describe the relations between data, chaotic tests will not be required. However, if this is not the case, investigating the main properties of chaoticity should not be disregarded.

Moreover, because it is based on the correlation dimension, the BDS test suffers from the same limitations. In particular, its performance depends on the size of data sets $(\mathrm{N})$ and $\varepsilon^{15}$, even though Brock et al. (1991) showed how the statistics of this test are correctly approximated in finite samples if

- the number of data $\mathrm{N}$ is greater than 500 .

- $\varepsilon$ lies between $0.5 \theta$ and $2 \theta$, where $\theta$ is the standard deviation of the series.

- the embedding dimension $\mathrm{m}$ is lower than $\mathrm{N} / 200$.

Moreover, it has been found that the BDS test has low power against certain forms of nonlinearity, such as self-exciting threshold AR processes and neglected asymmetry in volatility (Kuan 2008).

\subsection{Lyapunov Exponents}

The time series analysis tools described above-the BDS test and the correlation dimension - allow for the distinction between nonlinear systems with a certain degree of complexity and those without, relying on specific features of these systems: nonlinearity for the BDS test and fractal dimension for the correlation dimension. Therefore, considering the fact that the BDS test produces indirect evidence of nonlinear dependence, which is necessary but not sufficient for chaos (Barnett et al. 1995, 1997, Barnett and Hinich, 1992), and even though a nonlinear dynamic is low-dimensional, it cannot be considered chaotic; thus, we need a more appropriate tool to detect chaotic behaviour. The Lyapunov exponent may provide a more useful characterisation of chaotic systems because unlike the correlation dimension, which estimates the complexity of a nonlinear system, it indicates a system's level of chaos.

\footnotetext{
${ }^{13}$ See Brock et al., 1996.

${ }^{14}$ See Filoll, 2001.

${ }^{15}$ To deepen this point see Kyrtsou et al. (2001)
} 
The Lyapunov exponent (L) investigates another different, and perhaps more specific, characteristic of chaotic systems: their sensitivity to initial conditions. Two points with arbitrarily close but unequal initial conditions will diverge at exponential rates. The trajectories remain within a bounded set if the dynamic system is chaotic.

In calculating the divergence of the trajectories, we are interested in identifying what is known as the greatest exponent or Lyapunov characteristic exponent ${ }^{16}$. This exponent measures average exponential divergence or convergence between trajectories that differ only in having an "infinitesimally small" difference in their initial conditions and remains well defined for noisy systems.

To estimate $\lambda$ from experimental or observational data, there are two classes of methods, both of which are based on reconstructing the space state by the delay coordinates methods. The direct methods ${ }^{17}$ proposed by Wolf et al. (1985) and Rosenestein et al. (1993) are based on the calculation of the growth rate of the difference between two trajectories with an infinitesimal difference in their initial conditions.

In Jacobian methods ${ }^{18}$, data are used to estimate the Jacobians of underlying processes, and $\lambda$ is calculated from these. Nychka et al. (1992) proposed a regression method similar in some respects to the Gencay and Dechert test (1992), which involves the use of neural networks to estimate the Jacobians and $\lambda$; it is known as the NEGM test. Some remarkable advantages of the Jacobian methods over the direct methods are their robustness to the presence of noise and their satisfactory performance in moderate sample sizes (Shintani and Linton, 2004).

The general idea on which those methods are based is to follow two nearby points and calculate their average logarithmic rate of separation.

Consider $x_{0}$ and $x_{0}^{\prime}$ as two points in the state space with distance $\left\|x_{0}-x_{0}^{\prime}\right\|=\delta_{x_{0}}<1$.

Here, $\delta_{x t}$ is the distance after $T$ iterations between two trajectories emerging from these points; thus,

$\delta_{x t} \approx \delta_{x_{0}} e^{\lambda T}$

where $\mathrm{T}$ is the iteration number and $\lambda$ is the maximal Lyapunov exponent, which measures the average rate of divergence or convergence of two nearby trajectories. This process of averaging is the key to calculating accurate values of $\lambda$ using small, noisy data sets.

In a system with attracting fixed points of periodic orbit, the distance $\delta x\left(x_{0}, t\right)$ diminishes asymptotically with time. If the system is unstable, the trajectories diverge exponentially for a while but eventually settle down. If the system is chaotic, $\delta x\left(x_{0}, t\right)$ behaves erratically.

\footnotetext{
${ }^{16}$ "[...] maximal Lyapunov exponent [...] is the inverse of a time scale and quantifies the exponential rate by which two typical nearby trajectories diverge in time. In many situations the computation of this exponent only is completely justified, [...]. However, when a dynamical system is defined as a mathematical object in a given state space, $[\ldots]$ there exist many different Lyapunov exponent as there are space dimensions", Kantz e Schreiber, 1997, p. 174.

${ }_{18}^{17}$ Some limitations of this methods are highlighted in Shintani and Linton, 2004

18 To obtain the Lyapunov exponent from observational data, Eckmann and Ruelle (1985) and Eckmann et al. (1987) proposed a method, known as the Jacobian method, which is based on nonparametric regression.
} 
Hence, it is better to study the mean exponential rate of divergence of trajectories from two initially close points using the following algorithm:

$\lambda=\lim _{T \rightarrow \infty} \frac{1}{T} \sum_{t=1}^{T} \ln \frac{\left|\delta x\left(x_{0}, t\right)\right|}{\left|\delta x_{0}\right|}$

The exponents can be positive or negative, but at least one exponent must be positive for an attractor to be classified as chaotic. In particular, if $\lambda<0$, the system converges to a stable fixed point or stable periodic orbits. A negative value of the Lyapunov exponent is characteristic of dissipative or non-conservative systems. If $\lambda=0$, the system is conservative and converges to a stable cycle limit. If $\lambda>0$, the system is unstable and chaotic. Nearby points, no matter how close, will diverge. Therefore, if the system is chaotic, it will at least have a positive Lypunov exponent ${ }^{19}$. In fact, one definition of chaotic systems is based on a positive Lyapunov exponent (Mayer-Kress, 1986, Deneckere and Pelikan, 1986, Wolf et al. 1985). Finally, if $\lambda=\infty$, the system is random.

\subsection{Topological methods}

The tools described thus far, which are based on detecting the metric and dynamical invariants of attractors, are highly sensitive to noise (Barnett and Serletis 2000). In particular, their applications often require large, clean data sets. Now, if we consider the data sets provided in economics, which are small and noisy, the possibility of detecting chaotic behaviours is very limited. To overcome these limits, the attention of researchers is redirected towards the topological tools that provide the basis for a new way of testing data for chaotic behaviour (Mindlin et1990, Mindlin and Gilmore 1992, Tufillaro et al. 1990).

Topological tools are characterised by the study of the organisation of the strange attractor ${ }^{20}$ and exploit an essential property of chaotic systems, i.e., the tendency of a time series to nearly, although never exactly, repeat itself over time. This property is known as the recurrence property. Topological methods feature close returns plots (McKenzie, 2001, Gilmore, 1993a,b, 1996, 2001) and recurrence analysis (Eckmann et al. 1987). This latter is based on embedded delayed coordinates, while the former is implemented without embeddings.

These techniques make it possible to reveal correlations in data sets that are not possible to detect in the original time series. They do not require assumptions on the stationarity of a time series or the underlying motion equations and have been successfully applied in the sciences to detect chaos in experimental data. Moreover, they are particularly applicable to economic and financial data because they work well on relatively small data sets (Faggini, 2007, 2011) and are robust against noise. They preserve the time ordering of data and provide information about the system that generated them.

\footnotetext{
${ }^{19}$ "[...] the magnitude of the exponent reflecting the time scale on which system dynamics become unpredictable", Wolf et al., 1985, p. 285.

${ }^{20}$ A strange attractor is the set of points towards which chaotic system converges
} 
Of course, this does not mean that these tools are without problems. First, the threshold term $\varepsilon$, which compares the data, is subjective. This is true even if, unlike the BDS test, this term can be varied without altering the qualitative nature of the observed pattern of close returns (McKenzie, 2001). Second, both metric and topological tests suffer with non-stationary data $^{21}$. However, the problem of non-stationarity, while a problem for both metric and topological tests, seems to be more stringent for the former because it does not maintain the time ordering of data. Moreover, for recurrence analysis, we must take into account all limitations concerning the procedure of embedding coordinates.

\subsection{Close returns test and recurrence analysis}

The close returns test consists of a two parts: a qualitative component that is a graphical representation of the presence of chaotic behaviours, the Close Return Plot (CRP), and a quantitative one that tests the null hypothesis that the data are IID against both linear and nonlinear alternatives. It exhibits the same performance as the BDS test. Unlike the methods based on the correlation dimension, this tool detects the recursive behaviour of chaotic time series.

Let $\left\{x_{t}\right\}$ be a time series whose trajectories are orbiting in the face space. If the orbit is one period, the trajectory will return to the neighborhood of $x_{t}$ after an interval equals 1 ; if the orbit is two periods, it will return after an interval equals 2 , and so on.

Therefore, if $x_{t}$ evolves near a periodic orbit for a sufficiently long time, it will return to the neighbourhood of $x_{t}$ after some interval $(\mathrm{T})$. The criterion of closeness requires that the difference $\left|x_{i}-x_{i+T}\right|$ be very small. Computing all differences $\left|x_{t}-x_{t+i}\right|$, where $t=(1 \ldots n)$ , $i=(1 \ldots n-1)$ and $n$ is the length of sample, the close return test detects the observations for which $\left|x_{i}-x_{i+T}\right|$ is smaller than a threshold value $\varepsilon$. In the $\operatorname{plot}^{22}$ (Fig. 5), the horizontal axis indicates the number of observations $t$, where $t=(1,2, \ldots, N)$, and the vertical axis is $i$, where $i=(1,2, \ldots N-1) .$,$N is the observation number. If this difference is smaller, it is coded$ black; otherwise, it is coded white.

If the data are IID, the distribution of black dots will be random. In the plot, no pattern is evident (Fig. 5). If the time series is deterministic, it is possible to observe horizontal line segments. More specifically, the short horizontal line is symptomatic of chaotic dynamics (Fig. 6).

\footnotetext{
${ }^{21}$ "This is especially a concern when testing data for emerging countries, which typically undergo significant regulatory changes in the development period", McKenzie, 2001, p. 40.

${ }^{22}$ Examples by Gilmore 1993a,b, 1996, 2001
} 


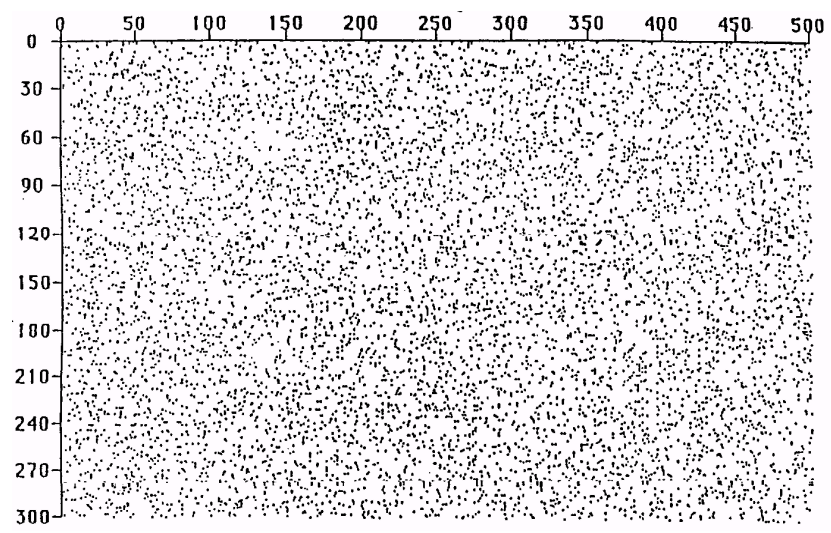

Fig. 5 Close returns plot of random data

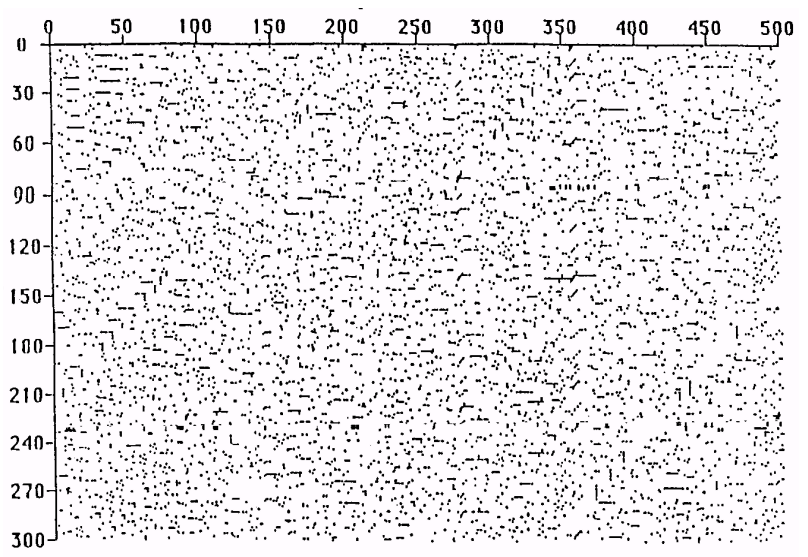

Fig. 6 Close returns plot series from logistic map

The second part of the test concerns the construction of a histogram that resumes the information of the close returns plot.

The histogram displays the number of close returns for each $i$, where $H_{i}=\sum \Theta\left(\varepsilon-\left|x_{t}-x_{t+i}\right|\right.$ and $\Theta$ is the Heaviside function. In the histogram, the chaotic data will show some peaks (Fig. 7a); otherwise, it will be uniformly distributed around some value $\bar{H}$ (Fig. 7b). 


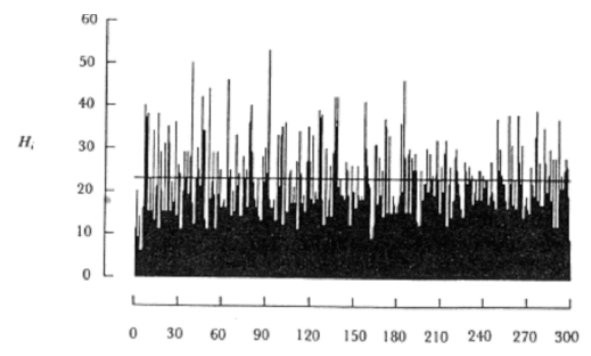

(a)

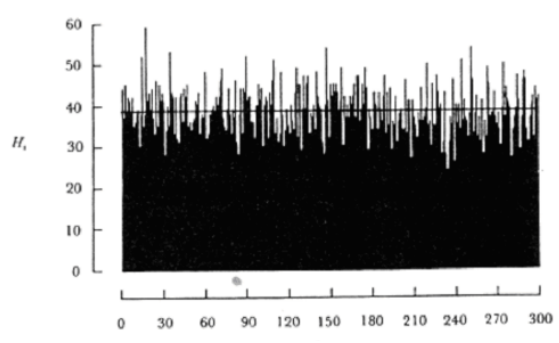

(b)

Fig. 7 Histogram of Henon series (a) and random series (b)

To define if on average $H_{i}=\bar{H}$ and to determine whether the null hypothesis of IID can be accepted, we have to calculate the chi-squared statistic:

$$
\chi_{t}^{2}=\frac{\sum_{i=1}^{k}\left[H_{i}-\bar{H}\right]^{2}}{n p}
$$

In Equation 12, $\bar{H}=n p$ and $i$ is equal to the number of observations over $p$, which is estimated in the following way:

$$
p=\frac{\text { total number of close returns }}{\text { total area of the plot }}
$$

Then the estimated $\chi_{t}^{2}$ is compared to the critical test value $\left(\chi_{c}^{2}\right)$ obtained with $(k-1)$ degrees freedom. If $\chi_{c}^{2}>\chi_{t}^{2}$, the null hypothesis that the data are IID is rejected (Gilmore 1993, 2001, MacKenzie 2001).

\subsection{Recurrence Analysis}

Like the close returns test, recurrence analysis consists of two parts: the recurrence plot (RP) developed by Eckmann et al. (1987), a graphical tool that evaluates the temporal and phase space distance, and recurrence quantification analysis (RQA) ${ }^{23}$, a statistical quantification of RP.

Recurrence analysis and the close returns plot are more similar because they are based on the same methodology but differ in the plot construction. Recurrence plots are symmetrical over the main diagonal. Moreover, while the close returns test analyses the time series directly and fixes a value $\varepsilon$ to estimate nearby points, the RP is based on the reconstruction of time series and an estimation of the points that are close. This closeness is measured by a critical radius so that a point is plotted as a coloured pixel only if the corresponding distance is below or equal to this radius. From the occurrence of lines parallel to the diagonal in the

\footnotetext{
${ }^{23}$ Zbilut J. P., Webber C. L., (1992).
} 
recurrence plot, it can be seen how fast neighboured trajectories diverge in phase space. The line segments parallel to main diagonal are points that move successively closer to each other in time and would not occur in a random as opposed to deterministic process. Chaotic behaviour produces very short diagonals, whereas deterministic behaviour produces longer diagonals. Thus, if the analysed time series is chaotic, then the recurrence plot shows short segments parallel to the main diagonal; on the other hand, if the series is white noise, then the recurrence plot does not show any kind of structure.

Nevertheless, sometimes the graphical output of RP is not easy to interpret because the signature of determinism, the set of lines parallel to the main diagonal, might not be so clear. As a consequence, Zbilut et al. $(1998,2000)$ proposed a statistical quantification of RP, which is well known as recurrence quantification analysis (RQA).

RQA defines the measures of the diagonal segments in a recurrence plot. These measures are recurrence rate, determinism, averaged length of diagonal structures, entropy and trend.

Recurrence rate (REC) is the ratio of all recurrent states (recurrence points percentage) to all possible states and is the probability of recurrence of a special state. REC is simply what is used to compute the correlation dimension of data. A recurrence plot can be considered to be a two-dimensional pictorial representation of the points that contribute to Eq. (6) for a particular value of $\varepsilon$.

Determinism (DET) is the ratio of recurrence points forming diagonal structures to all recurrence points. $\mathrm{DET}^{24}$ measures the percentage of recurrent points forming line segments that are parallel to the main diagonal. A line segment is a point's sequence, which is equal to or longer than a predetermined threshold. These line segments reveal the existence of deterministic structures, absence instead of randomness.

Maxline (MAXLINE) represents the averaged length of diagonal structures and indicates the longest line segments that are parallel to the main diagonal. It is claimed to be proportional to the inverse of the largest positive Lyapunov exponent. A periodic signal produces long line segments, while the noise does not produce any segments. Short segments indicate chaos.

Entropy (ENT) (Shannon entropy) measures the distribution of those line segments that are parallel to the main diagonal and reflects the complexity of the deterministic structure in the system. This ratio indicates the time series structuredness so high values of ENT are typical of periodic behaviours, while low values are typical of chaotic behaviours. A high ENT value indicates a large diversity in diagonal line lengths; low values indicate small diversity in diagonal line lengths ${ }^{25}$. "[...] short line max values therefore are indicative of chaotic behaviours" ${ }^{26}$. The value trend (TREND) measures the paling of the patterns of RPs away from the main diagonal (used for detecting drift and non-stationarity in a time series).

\footnotetext{
${ }^{24}$ "This is a crucial point: a recurrence can, in principle, be observed by chance whenever the system explores two nearby points of its state space. On the contrary, the observation of recurrent points consecutive in time (and then forming lines parallel to the main diagonal) is an important signature of deterministic structuring" Manetti et al. (1999)

${ }^{25}$ Trulla et al. 1996

${ }^{26}$ Iwanski and Bradley (1998), Atay and Altintas (1999)
} 
An extensive survey of different software used to apply these techniques is provided in Belaire-Franch and Contreras (2002).

\section{Evidence of Chaos in Economic Data}

In this section, we will summarise the main results obtained by applying chaos tests to economic and financial data. We will distinguish between the applications to macroeconomic data and financial data because of the variation in the results between the two.

Of course, this survey is not meant to be exhaustive but only indicative of the state of the art. In applying the BDS test to the residuals of linear models, Sayers (1986) and Frank and Stengos (1987) rejected the presence of chaos in the data of work stoppages and Canadian macroeconomic series, respectively. Brock and Sayers (1988) conducted their analysis using U.S. macroeconomic data ${ }^{27}$ and showed the presence of nonlinearity but presented weak evidence of chaos. Barnett and Chen (1988) demonstrated the presence of chaos in the U.S. Divisia monetary aggregates. The estimated value of the correlation dimension reaches a saturation point between 1.3 and 1.5 , indicating the presence of a chaotic attractor in a bidimensional face space. In Frank et al. (1988), the application of the correlation dimension to residuals of the AR model and Lyapunov exponent did not show the presence of chaos in macroeconomic data from 1960 to 1988 for West Germany, Italy, Japan and England. DeCoster and Mitchell (1991), in applying the correlation dimension and the BDS test to weekly monetary variables (Divisia M2 and M3), showed that nonlinear and even chaotic monetary dynamics for U.S. data cannot be dismissed. The same procedure and conclusions were reported by Frank and Stengos (1989). They investigated daily prices from the mid1970s to the mid-1980s for gold and silver using the correlation dimension and Kolmogorov entropy. They found that the correlation dimension is between 6 and 7 and that the Kolmogorov entropy is about 0.2 for both assets.

Different conclusions were reported by Yang and Brorsen (1992). They did not find chaos in the daily prices of some agricultural commodities or in several futures markets, including those of gold and silver. The evidence of nonlinearity is not consistent with chaos because the shuffled data do not satisfy the saturation condition of correlation dimension. Cromwell and Labys (1993) apply the correlation dimension and the Lypunov exponent to commodity prices for the period from 1960 to 1992 . They find chaos in daily prices of corn but not in those of sugar, coffee and cacao.

In Chavas and Holt (1991), the application of the correlation dimension, the BDS test, and the Lyapunov exponent provided clear evidence that the dynamic process generating the pork cycle is nonlinear, even if the evidence in favour of chaos is less conclusive. These results are confirmed by Streips (1995), who by applying the same tests on observations of the monthly U.S. hogcorn price ratio for the period 1910-94, shows that data are chaotic.

Kohzadi and Boyd (1995) tested for the presence of chaos and nonlinear dynamics in monthly cattle prices for the period 1922-90. The Grassberger and Procaccia, BDS and

\footnotetext{
${ }^{27}$ Employment 1950-I to 1983-IV, unemployment 1949-I to 1982-IV, monthly post-war industrial production, and pig iron production 1877-1937.
} 
Hurst Exponent tests showed evidence of chaos in these data. Bajo-Rubio et al. (1992) presented estimates of the correlation dimension and the largest Lyapunov exponent for daily data regarding the Swedish Krona vs. the Deutsche Mark, ECU, U.S. Dollar and Yen exchange rates. They find indications of deterministic chaos in all exchange rate series. However, because of the limited number of data (1985-1991), the estimates for the largest Lyapunov exponents are not reliable, except in the Swedish Krona-ECU case where they find a low-order chaotic behaviour.

The close returns test has been implemented in detecting and visualising chaotic behaviour in macroeconomic time series using some monetary aggregates. Montoro and Paz (1997) did not find evidence of chaos in the Divisia M2 series, in contrast with the results obtained in several previous works.

Monthly aggregate air transport service series for over two decades have been analysed using BDS test by Adrangi et al. (2001a). While strong evidence of nonlinearity is found in the data, this evidence is not consistent with chaos. The ARCH-type model explains the nonlinearity in the data well.

The daily oil products for the Rotterdam and Mediterranean petroleum markets have been tested for the presence of chaos and nonlinear dynamics by Panas and Ninni (2000). The correlation dimension, BDS test and Lyapunov exponents show strong evidence of chaos in a number of oil products: Naphtha, Mogas Prem, Sulfur FO 3.5\% e FO 1.0\%, Gasoil e Mogas REG.UNL. Moreover, Panas (2002) investigated the price behaviour in the London Metal Exchange market using the two most attractive nonlinear models: long memory and chaos. The results indicate that the dynamics of the LME market can be attributed to long memory (aluminium and copper), i.e., a persistent process exhibiting self-similarity, shortmemory behaviour (nickel and lead price returns), antipersistent (or intermediate memory in the case of zinc returns) and a deterministic chaotic process (in the case of tin returns).

Serletis and Shintani (2006) find statistically significant evidence against low-dimensional chaos in Canadian and US data.

Barkoulas (2008) applied both metric and topological methodologies to test for deterministic chaotic structure in simple-sum and Divisia monetary aggregates. The results did not satisfy any of the three indications of chaos. The monetary dynamics are not chaotic. Faggini (2007, 2011), starting from the conclusion by Frank et al. (1988) that refused the chaotic hypothesis in macroeconomic data, applied visual recurrence analysis to the same time series and found chaos in GNP data from Japan and United Kingdom.

\section{Evidence of Chaos in Financial data}

The apparent randomness of financial markets led some economists to become interested in chaos theory as a theoretical framework able to explain those fluctuations. One of the first applications of chaos tests to financial data was carried out by Scheinkman and LeBaron (1989). They analysed the United States weekly returns on the Center for Research in Security Prices (CRSP), applying the BDS test on residuals of linear models. They found rather strong evidence of nonlinearity and some evidence of chaos. This is because the correlation dimension of the shuffled residuals appeared to be much greater than that of the original residuals. That is, it does not reach a saturation point, indicating the completely 
random behaviour of the shuffled data. If time series data are chaotic, the estimated correlation dimension of residuals is the same as that of the original data. When the data are stochastic, the correlation dimension of the residuals increases as the embedding dimension increases. The residuals are less structured than the original data.

The same conclusion was reached by Hsieh (1991), who uses the BDS test to detect chaos in weekly stock returns data from the Center for Research in Securities Prices from 1963 to 1987. All of the data were first filtered by an autoregression model. The correlation dimension technique was used by DeCoster et al. (1992) to find evidence of chaos in daily sugar, silver, copper, and coffee futures prices for the period 1960-1989.

Vaidyanathan and Krehbiel (1992) and Mayfield and Mizrach (1992) found evidence of chaos behaviour in the S\&P 500 index, though this conclusion is based on the results of correlation dimension rather than on Lyapunov exponent estimates.

In Blank (1991), the estimates of correlation dimensions and Lyapunov exponents on soybean and S\&P 500 futures prices are consistent with the presence of deterministic chaos. Yang and Brorsen (1993) found evidence of nonlinearity in several futures markets, which is consistent with deterministic chaos in about half of the cases. Hsieh (1993) found evidence of nonlinearity in four currency futures contracts, but found that nonlinearity is the result of predictable conditional variances. In Brorsen and Yang (1994), nonlinear dependence is not removed for the value-weighted index or the S\&P 500 stock index. Standardised residuals from the GARCH model are not IID for two of three returns series. The application of BDS test proves that deterministic chaos cannot be dismissed.

Abhyankar et al. (1995) test for the presence of nonlinear dependence and chaos in real-time returns on the U.K. FTSE-100 Index (about 60.000 data points). Their results suggest that GARCH can explain some but not all of the observed nonlinear dependence. The application of Hinich test (1982), BDS test, and Lypunov estimates fail to detect chaos in the data.

Sewell et al. (1996) examined weekly changes for the period 1980 to 1994 in six major stock indices (the US, Korea, Taiwan, Japan, Singapore and Hong Kong) and the World Index as well as the corresponding foreign exchange rates between the US and the other five countries. They concluded that ' $[\mathrm{t}]$ hese results do not prove the existence of chaos in these markets but are consistent with its existence in some cases'. Abhyankar et al. (1997) and Serletis and Shintani (2003) reject the null hypothesis of low-dimensional chaos in the S\&P 500 and Dow Jones Industrial Average.

Serletis and Gogas (1997) tested for chaos in seven East European black market exchange rates by applying the BDS test, the NEGM test (Nychka et al., 1992), and the Lyapunov exponent. They found evidence consistent with a chaotic nonlinear generation process in two out of the seven series: the Russian ruble and East German mark. In particular, the BDS test rejects the null hypothesis of IID for three of the seven markets, whereas the Lyapunov exponent estimator proposed by Nychka et al. (1992) supports the hypothesis of chaotic dynamics in two markets. Barkoulas and Travlos (1998) investigated the existence of a deterministic nonlinear structure in the stock returns of the Athens Stock Exchange (an emerging capital market) and found no strong evidence of chaos.

Gao and Wang (1999) examined the daily prices of four futures contracts (S\&P 500, JPY, DEM and Eurodollar) and found no evidence of deterministic chaos. Andreou et al. (2000) 
examined four major currencies against GRD and found evidence of chaos in two out of four cases.

Adrangi et al. (2001b), implementing the BDS test, correlation dimension and Kolmogrov entropy, investigated the presence of low dimensional chaos in crude oil, heating oil and unleaded gasoline future price from the early 1980s. They find strong evidence of nonlinearity inconsistent with chaos.

Gilmore (2001) implemented the close returns test to examine chaos presence in some foreign exchange rates. The results did not support chaotic explanations without excluding other possible forms of nonlinear structure (Gilmore, 1993a and 1993b). The test was implemented on the residuals of some ARCH-type filters as well, and the results have indicated that the models have captured some, although not all, of the nonlinear dependence among data. Additionally, McKenzie (2001) investigated the presence of chaos in a wide range of major national stock market indices using the close return test. The results indicate that the data are not chaotic, although considerable nonlinearities are present.

Daily data for the Swedish Krona against the Deutsche Mark, the ECU, the US Dollar and the Yen exchange rates are tested by Bask (2002) using the Lypunov exponent. In most cases, the null hypothesis that the Lyapunov exponent is zero is rejected in favour of a positive exponent.

Shintant and Linton (2004) use 18.490 daily observations of the Dow Jones Industrial Average from January 1928 to October 2000. They used the Lyapunov exponent estimator proposed by Nychka et al. (1992). The results did not indicate chaotic behaviours in the data. Foreign exchange rates vs. IRR (Iranian Rial) have been investigated by Torkamani et al. (2007), who show that the data in this market have complex chaotic behaviour with a large degree of freedom. The tests applied are the correlation dimension and the Lyapunov exponent.

Antoniou and Vorlow (2005) investigated the 'compass rose' patterns revealed in phase portraits (delay plots) of FTSE 100 stock returns and found a strong nonlinear and possibly deterministic signature in the data-generating processes

The nonlinearity and chaoticity of exchange rate time series are investigated by Liu (2009). The BDS test and surrogate data method indicated that the exchange rate time series of Canadian Dollars to United States Dollar (CD/USD), Japanese Yen to United States Dollar (JY/USD) and United States Dollar to British Pound (USD/BP) exhibit nonlinearity, while the exchange rate time series of United States Dollar to EURO (USD/EURO) is linear. The largest positive Lyapunov exponents have provided evidence for the possibility of deterministic chaos in the daily exchange rate time series of $\mathrm{CD} / \mathrm{USD}, \mathrm{JY} / \mathrm{USD}$ and $\mathrm{USD} / \mathrm{BP}$.

Das and Das (2007) implemented the Lyapunov exponent and surrogate data method to investigate the chaoticity of Foreign Exchange Rates of several countries. They found an indication of deterministic chaos in all exchange rate series

Adrangi et al. (2010), employing the daily bilateral exchange rates of the dollar, conducted a battery of tests for the presence of low-dimension chaos (correlation dimension tests, BDS tests, and tests for entropy). The strong evidence of nonlinear dependence in the data is not 
consistent with chaos. The nonlinear dependencies in the dollar exchange rate returns series arise from the GARCH-model rather than from a chaotic structure.

More recently, the rate of returns series for six Indian stock market indices are tested for chaos (Mishra 2011). The results from the test of independence on filtered residuals suggest that the existence of nonlinear dependence, at least to some extent, can be attributed to the presence of conditional heteroskedasticity. To account for the remaining nonlinearity in the data, the Lyapunov exponent is estimated ${ }^{28}$. The result is a positive value in two out of six cases, indicating that these return series are generated by a chaotic system.

Analyses on financial data are performed by recurrence analysis in Mizrach B., (1996), McKenzie M D., (2001), Holyst J. A., et al. (2000-2001), Strozzi F., et al. (2002). BelaireFranch et al. (2001) analyse (RP e RQA) the exchange rates of 16 OECD countries from 1957 to 1998. After a filtering procedure with the ARMA model, RQA applied on residuals shows the presence of chaotic dynamics in the data.

Daily index prices, consisting of free float-adjusted market capitalisation stock indices of developed and emerging markets between January 1995 and December 2009, are analysed by Bastos and Caiado (2011) using recurrence analysis. The statistical tests suggested that the dynamics of stock prices in emerging markets characterised by higher values of RQA measures when compared to their developed counterparts.

\section{Is there chaos in economic and financial data?}

Although the literature on tests for chaos in economic and financial time series is voluminous, there are no uncontroversial results to speak of. Clearly, the short review presented above suggests that there is ample evidence of the presence of nonlinearities and some evidence of deterministic chaos.

The difficulty of using chaos theory in economics is a direct consequence of some problems related to the application of these techniques to economic data.

First of all, data quantity and data quality are crucial when applying these techniques, and the main obstacle in empirical economic analysis is addressing short and noisy data sets. Data quantity and data quality in economics constitute a significant obstacle in chaotic-economic theory.

Moreover, testing macroeconomic series is regarded with some suspicion; not only are the gathered data insufficient to perform tests, but the macro time series also involve mixed effects: it is not only the distinction between noise and nonlinearities that must be determined but also the eventual source of nonlinearity; they are usually aggregated and derived from a system whose dynamics and measurement probes may be changing over time. Granger (2001) shows that the aggregation of independent series hides the nonlinear or chaotic signals. Current tests used to detect chaotic structure often fail to find evidence of

\footnotetext{
${ }^{28}$ It is clearly observable that in 2 out of 7 cases the point estimates of Lyapunov exponents are positive. This implies that the returns of Bank Nifty and CNX IT exhibit a chaotic nonlinear generating process, and therefore, the nonlinear structure in these return series is possibly deterministic in nature. Further, a negative Lyapunov exponent (i.e. in the case of CNX NIFTY, BSE SENSEX, BSE 200 and BSE 100) indicates that the nature of the series is consistent with a stochastic process rather than a deterministic low-dimensional chaotic system.
} 
chaos in aggregated data, even if those data are generated by a nonlinear deterministic process.

Little or no evidence for chaos has been found in macroeconomic time series. Investigators have found substantial evidence for nonlinearity but relatively weak evidence for chaos per se. This is due to the small sample sizes and high noise levels for most macroeconomic series. In contrast to laboratory experiments, through which a large number of data points can be easily obtained, most economic time series consist of monthly, quarterly, or annual data, with the exception of some high-frequency financial series. In fact, the analysis of financial time series has led to results that are, as a whole, more reliable than those of macroeconomic series. This is because financial time series data are available in large quantities over many disaggregated time intervals, though this literature is not free of controversial results.

Controversial results also arise from using inappropriate analytical methodologies that are more similar to standard statistical protocol. To distinguish between chaotic and non-chaotic behaviours, all researchers, before applying chaos tests, filtered the data using either linear or nonlinear models (Frank and Stengos 1989, Blank 1991, Cromwell and Labys 1993, Yang and Brorsen 1992, 1993), in most cases, using ARCH-type models. When these do not capture all of the nonlinearities in the economic and financial data (Hsieh, 1991,Vaidyanathan and Krebhiel 1992), chaos analysis is conducted on the residuals. This procedure has been used by Frank and Stengos (1989), DeCoster et al. (1992) Chavas and Holt (1991) and Bask (2002), among others.

The filtering procedure was supported by Brock (1986), who stated that before testing for a possible nonlinear dependency among the observations, we need to remove all linear correlations that may cause the null hypothesis to be rejected. He also argued that with an infinite amount of noise-free data, possible nonlinear structures should be unaffected by the implementation of a linear filtering process. Removing all linear structure is difficult, but a good approximation can be achieved by using an autoregressive moving average (ARMA) fit to stationary data. With the assumption that the residuals are filtered for linear dependence, it is reasonable to assert that any resulting dependence found in the residuals must be nonlinear. Then when nonlinearity is found, ARCH-type models are applied to detect the source. If unexplained nonlinearity remains, chaos tests are applied. In this assertion by Brock (1986), we must highlight the fact that the linear filtering procedure is irrelevant if the data are infinite, noise-free, and stationary ${ }^{29}$, conditions that are not testable for economic and financial data.

More generally, the open question is whether the chaotic properties of a process are invariant to linear and nonlinear transformations. It has been proved that linear and nonlinear filters can distort potential chaotic structures (Chen 1993, Wei and Leuthold 1998) and may affect the dimensionality of the original data (Chen 1993, Panas and Ninni 2000, Panas 2002), providing a false indication of chaos. Chen (1993) shows that the correlation dimension is

\footnotetext{
${ }^{29}$ Stationarity is an important property of data because in the stationary time series the statistical properties do not change over time.
} 
invariant to filtering by the MA (moving average model) because, in this way, the fractal structure of the dynamics is lost.

Moreover, sometimes the conclusions both for and against chaos are reached by applying only one type of chaos test. For example, Kohzadi and Boyd (1995) find chaos by using only the BDS test and R/S analysis.

To produce convincing results, we have to employ all tests for chaos to exploit their different potentials and limits. Few published papers have jointly applied the BDS test, the correlation dimension test, and the test for a positive Lyapunov exponent. Very few use topological tools (Barkoulas, 2008).

The consequence of this resonates in the words of Granger and Terasvirta (1992): "Deterministic (chaotic) models are of little relevance in economics and so we will consider only stochastic models". The question was intensified by Jaditz and Sayers (1993), who reviewed a wide variety of data to conclude that there was no evidence for chaos, though they did not deny the indication of nonlinear dynamics of some sort. Moreover, controversies are also produced by the nature of the tests themselves. There may be very little robustness of such tests across variations in sample size, test methods, and data aggregation methods. It is widely known that problems related to the quality and lack of sufficient amounts of data, the issue of appropriate level of disaggregation, and the proper definition of methods for detecting white noise create considerable obstacles to constructing a meaningful and coherent statistical theory about the dynamics of economic and financial data. Following the studies so far discussed, we have to admit that no natural deterministic explanation exists for the observed economic fluctuations that are produced by external shocks or by inherent randomness and, consequently, an inherent unpredictability

Moreover, up until now, we have been interested only in low-complexity chaotic behaviour. The failure to detect low-dimensional chaos does not preclude the possibility of there being high-dimensional chaos in these variables (Day 1994). It is possible that the underlying nonlinear structure of the economy is more complex and that the chaotic dynamics it exhibits are of a higher dimensionality. The algorithms presented above were developed to detect chaos in experimental data. Because physicists can often generate very large samples of high-quality data form laboratory experiments, they find these algorithms to be directly applicable to their research. Consequently, further theoretical advances are required to develop tests that are able to detect more complex forms of chaotic behaviours.

\section{Conclusions}

Researchers in economics and finance have been interested in testing for nonlinear dependence and chaos for almost two decades now. A wide variety of reasons for this interest have been suggested, including an attempt to improve the forecasting accuracy of linear time series models and to better explain the dynamics of the underlying variables of interest using a richer class of models than that permitted by limiting the set to the linear case.

After an exuberant flurry of publications, the search for chaos in economics has been gradually becoming less enthusiastic over the last two decades, as no empirical support for the presence of chaotic behaviours in economics has been found. The literature described 
above does not provide a solid support for chaos as a consequence of the high noise level that exists in most aggregated economic time series, the relatively small sample sizes of data, and the weak robustness of chaos tests for these data.

To summarise, surely there is no reason to suppose that the economy is linear (George and Oxley, 2007), so we should be able to find evidence of nonlinearity in economic data.

Therefore, if we prove that the data are chaotic, we will be able to prove that there is a deterministic system that generates them. This could be a big step forward in clarifying the "nature" of the economy.

Chaotic nonlinear systems can endogenise shocks. Chaotic dynamic models allow for the explanation of persistent and irregular fluctuations without stochastic exogenous shocks introduced ad hoc.

Most economic variables, whether micro-level, such as prices and quantities, or macro-level, such as consumption, investment and employment, oscillate. $t$ is difficult to find a specific pattern in these oscillations at the level of micro and macro variables because they are not cyclic (Day, 1994) and not due to external shocks.

Moreover, if the economy is chaotic, then we can create a complete and closed model for it. This development would significantly help forecasting and control efforts in the short run. Being able to detect chaos in economic data is the first condition that must be met to apply a chaotic control to phenomena that generate the data. In fact, chaos theory offers attractive possibilities for control strategies (Faggini 2008, 2009), which seems particularly relevant to gaining insights into economic policies. Using sensitivity to initial conditions to move from given orbits to other orbits of attractors means choosing different behaviours of systemsthat is, a different trade-off of economic policy. Moreover, the employment of an instrument of control in terms of resources to achieve a specific goal of economic policy will be smaller compared to the use of traditional techniques of control.

Thus, even if we agree with Barnett (2006) that "...the economics profession, to date, has provided no dependable empirical evidence of whether or not the economy itself produces chaos, and I do not expect to see any such results in the near future. The methodological obstacles in mathematics, numerical analysis, and statistics are formidable", we do not have the slightest idea of whether or not the economy exhibits chaotic nonlinear dynamics, and hence, we are not justified in excluding the possibility. Moreover, part of the controversy and the resulting conclusions are due to the misconception that low-dimensional chaos can be expected to be present generically in all economic phenomena. We cannot assume deterministic chaos for any measured time series.

The results of chaos tests do not prove the existence of chaos in all economic variables but are consistent with its existence; in some cases, this could mean only that some economic phenomena are less complex than others and that the economy of a country or simply a single market of an economy is chaotic, not that an economy is as a whole is chaotic. Given these considerations, studies in this area should grow both in size and importance as a field of their own within economics, although the empirical task of extracting evidence of chaotic dynamics from economic time series is objectively more difficult than in the natural sciences. 
We have to create techniques that acknowledge that a basic difference exists between physics, which is generally an exact science, and economics. To date, the application of chaos theory has been only a mechanical transfer that has not taken into account the specific features of economic systems. Surely, compared with neoclassical theory models, chaos theory in economics allows researchers to interpret phenomena considered non-influential, exogenous, and stochastic.

\section{References}

Abhyankar A. H., Copeland L. S., Wong W., (1995), Non-linear dynamics in real-time equity market indices: evidence from the UK, Economic Journal, vol. 105, pp. 864-880.

Abhyankar A. H., Copeland L. S., Wong W., (1997), Uncovering nonlinear structure in realtime, stock-market indexes: the S\&P 500, the DAX, the Nikkei 225, and the FTSE-100, Journal of Business and Economic Statistics, vol. 15, pp. 1-14.

Adrangi B., Allender M., Chatrath A., and Raffiee K., (2010) Nonlinear Dependencies And Chaos In The Bilateral Exchange Rate Of The Dollar, International Business \& Economics Research Journal vol.9, Number 3

Adrangi B., Chatrath A., and Raffiee K., (2001a) The demand for US air transport service: a chaos and nonlinearity investigation, Transportation Research Par, E 37, pp 337-353.

Adrangi B., Chatrath A., Dhanda K.K., Raffiee K., (2001b) Chaos in oil prices? Evidence from futures markets, Energy Economics, n ${ }^{\circ} 23$ (2001) pp.405-425.

Andreou A. S., Pavlides G., and Karytinos A., (2000). Nonlinear Time- Series Analysis of the Greek Exchange-Rate Market. International Journal of Bifurcation and Chaos, vol. 10(7), pp. 1729-1758.

Antoniou A., Vorlow C. E., (2005). Price Clustering and Discreteness: Is there Chaos behind the Noise? Physica A, vol. 348, 389-403.

Atay F. M., Altintas Y., (1999), Recovering smooth dynamics from time series with the aid of recurrence plots, Physical Review E, vol. 59, pp. 6593-6598.

Bajo-Rubio O., Fernández-Rodríguez F., Sosvilla-Rivero S., (1992), Chaotic Behavior in exchange-rate series. First result for the Peseta-U.S. Dollar case, Economics Letters, vol. 39, pp. 207-211.

Barkoulas J. T., (2008) Testing for deterministic monetary chaos: Metric and topological diagnostics, Chaos, Solitons and Fractals, 38, pp. 1013-1024.

Barkoulas J., Travlos N., (1998) Chaos in an Emerging Capital Market? The Case of the Athens Stock Exchange, Applied Financial Economics, 8(3), pp. 231-243.

Barnett W. A., (2006) Comments on "Chaotic monetary dynamics with confidence, Journal of Macroeconomics, 28, pp. 253-255.

Barnett W. A., Serletis A., (2000) Martingales, nonlinearity, and chaos, Journal of Economic Dynamics \& Control, 24, pp. 703-724.

Barnett W., He Y., (2000), Unsolved Econometric Problems in Nonlinearity, Chaos, and Bifurcation, Economics Working Paper Archive at WUSTL, n. 09-06.

Barnett W.A., Hinich M.J., (1992). Empirical chaotic dynamics in economics .Annals of Operations Research, 37, 1-15. 
Barnett W.A., Chen P., (1988), The Aggregation Theoretic Monetary Aggregates are Chaotic and Have Strange Attractors: An econometric application of mathematical of chaos, in Barnett W. a., Berndt E., White H., (eds) Dynamic Econometric Modeling, Proc. 3rd International Symposium on Economic Theory and Econometrics, Cambridge University Press.

Barnett, W.A., Gallant, A.R., Hinich, M.J., Jungeilges, J., Kaplan, D. \& Jensen, M.J. (1995). Robustness of nonlinearity and chaos test to measurement error, inference method, and sample size. Journal of Economic Behavior and Organization, 27, 301-320.

Barnett, W.A., Gallant, A.R., Hinich, M.J., Jungeilges, J., Kaplan, D, and Jensen, M.J. (1997). A single-blind controlled competition between tests for nonlinearity and chaos. Journal of Econometrics

Barnsley M., (1988), Fractals Everywhere, Boston Academic Press.

Bask M., (2002), A Positive Lyapunov Exponent in Swedish Exchange Rates, Chaos, Solitons and Fractals, vol. 14, iss. 8 pp. 1295-1304.

Bastos J. A., Caiado J., (2011) Recurrence quantification analysis of global stock markets, Physica A: Statistical Mechanics and its Applications, 390, pp. 1315-1325.

Belaire-Franch J, Contreras D., (2002), Recurrence Plots in Nonlinear Time Series Analysis: Free Software, Journal of Statistical Software, 7(9) http:// aeser.anaeco.uv.es/pdf/dt/dt0101.pdf

Belaire-Franch J., Contreras-Bayarri D., Tordera-Lledó L., (2001), Assessing Non-linear Structures in Real Exchange Rates Using Recurrence Plot Strategies, Physica D, vol. 171, pp. 249-264.

Benhabib J., Day R., (1981) Rational choice and erratic behaviour, Review of Economic Studies, vol. 48, pp. 459-472.

Blank S. C., (1991), Chaos in Futures Markets: A Nonlinear Dynamically Analysis, The Journal of Futures Markets, vol. 11, pp. 711-728.

Bollerslev T., (1986), Generalized autoregressive conditional heteroscedasticity, Journal of Econometrics, vol. 31, pp. 307-327.

Brock W. A., Hsieh D.A., Le Baron B., (1991), Non linear dynamics, chaos, and instability: statistical theory and economic evidence, MIT Press, Cambridge, MA.

Brock W.A., Dechert W.D., Scheinkman J., (1987), A Test for Independence Based on the Correlation Dimension, Madison: Department of Economics, University of Wisconsin, SSRI Working Paper 8702.

Brock W.A., Dechert W.D., Scheinkman J., (1996) A test for independence based on the correlation dimension, Econometric Reviews, vol. 15, pp. 197-235.

Brock W.A., Sayers C., (1988), Is the business cycle characterized by deterministic chaos? Journal of Monetary Economics, 22.1, pp. 71-90.

Brock WA., (1986), Distinguishing random and deterministic system, Journal of Economic Theory, $\mathrm{n}^{\circ}$ 40(1), pp. 68-195.

Brorsen B.W., Yang S., (1994), Nonlinear dynamics and the distribution of daily stock index returns, The Journal of Financial Research, vol. 17, pp. 187-203.

Caputo E., Sello S., V. Marcolongo, Nonlinear Analysis of Experimental Noisy Time Series in Fluidized Bed Systems, Submitted to: Chaos Solitons and Fractals. 
Chavas J., Holt M., (1991), On Nonlinear Dynamic: The Case of Pork Cycles, American Journal of Agricultural Economics, vol. 73, pp. 819-828.

Chen P., (1993), Searching for Economic Chaos: A Challenge to Econometric Practice and Nonlinear Tests, Day R. H., Chen P., (eds), Nonlinear Dynamics and Evolutionary Economics, pp. 217-253, Oxford University Press.

Cheng B., Tong H., (1992), On consistent nonparametric order determination and chaos, Journal of the Royal Statistical Society, Series B, Methodological, 54, pp. 427-449.

Cromwell J.B., Labys W. C., (1993), Testing for Nonlinear Dynamics and Chaos in Agricultural Commodity Prices, Memo Institute for Labor Study, West Virginia University. Cutler C. D., (1991), Some results on the behaviour and estimation of the fractal dimensions of distributions on attractors, Journal of Statistical Physics, vol. 62, pp. 651-708.

Das A., Das P., (2007) Chaotic analysis of the foreign exchange rates, Applied Mathematics and Computation, $\mathrm{n}^{\circ} 185$ pp. $388-396$.

Day R. H., (1982), Irregular growth cycles, American Economic Review, vol. 72, pp. 406414.

Day R. H., (1994), Complex Economic Dynamics, vol 1. An introduction to Dynamical Systems and Market Mechanisms, Cambridge, MA: MIT Press

DeCoster, G. P., Labys W. C., Mitchell D. W., (1992), Evidence of Chaos in Commodity Futures Prices, The Journal of Futures Markets, vol. 12, pp. 291-305.

DeCoster, G. P., Mitchell, D. W., (1991) Nonlinear Monetary Dynamics, Journal of Business \& Economic Statistics, 9, pp. 455-461.

DeLima P. J. F., (1998) Nonlinearities and nonstationarities in stock returns, Journal of Business Economic Statistics, 16, pp. 227-236.

Deneckere R., Pelikan S., (1986) Competitive Chaos, Journal of Economic Theory, vol. 30, pp. 13-25.

Devaney R. L., (1986), An Introduction to Chaotic Dynamical Systems, Adison-Wesley, New York.

Ditto W., Munakata T., (1995) Principles and Applications of Chaotic Systems, Communication of the ACM, Emerging Technologies, vol. 38, n. 11, pp. 96-102.

Eckmann J. P., Ruelle D., (1985), Ergodic Theory and Strange Attractor, Review of Modern Physics, vol. 57, pp. 617-656.

Ekmann J. P., Kamphorst S. O., Ruelle D., (1987), Recurrence Plots of Dynamical Systems, Europhysics Letters, 4, 9, pp. 973-977.

Engle R.F., (1982) Autoregressive conditional heteroskedasticity with estimates of the variance of United Kingdom inflation, Econometrica 50, 987-1007.

Faggini M., (2007), Visual Recurrence Analysis: an Application to Economic Time Series, in Complexity Hints for Economic Policy, M. Salzano e D. Colander (eds.), Springer.

Faggini M., (2008) Chaotic Systems and New Perspectives for Economics Methodology. A Review from Literature (2008), in Complexity and Policy Analysis: Tools and Concepts for Designing Robust Policies in Complex World, Richardson, K. A., Dennard, L., \& Morcol, G. (eds.), ISCE Publishing, Goodyear.

Faggini M., (2009) Chaos and Chaotic Dynamics in Economics, Nonlinear dynamics, Psychology and Life Sciences (2009), 13(3), pp. 327-40. 
Faggini M.,(2011) Chaos Detection in Economics. Metric versus Topological Tools, MPRA Paper No. 30928.

Falconer K., (1990), Fractal Geometry, Chicester, Wiley.

Farmer J. D., Ott E., Yorke J. A., (1983), The dimension of chaotic attractor, Physica D, vol. 7, pp. 153-180.

Fillol J, (2001) Limits of the Tools for Detection of chaos in Economy. Application to the stock returns MODEM, University of Paris X-Nanterre, http://www.univorleans.fr/DEG/GDR ecomofi/Activ/fillolpau.pdf.

Frank M., Gencay R., Stengos T., (1988), International Chaos, European Economic Review, vol. 32, pp. 1569-1584.

Frank M., Stengos T., (1987), Some evidence concerning macroeconomic chaos, Discussion paper $\mathrm{n}$. 1987-2, University of Guelph.

Frank M., Stengos T., (1988), Chaotic Dynamics in Economic Time Series, Journal of Economic Surveys, vol. 2 n ${ }^{\circ}$ 2, 103-133.

Frank M., Stengos T., (1989) Measuring the Strangeness of Gold and Silver Rates of Return, Review of Economic Studies, vol. 56, pp. 553-567.

Gao A. H., Wang G. H. K., (1999). Modeling Nonlinear Dynamics of Daily Futures Price Changes. The Journal of Futures Markets, vol.19(3), 325-351.

Gençay, R., W. D. Dechert (1992). An algorithm for the n Lyapunov exponents of an ndimensional unknown dynamical system, Physica $D, 59$, pp-142-157.

George D., Oxley L. T., (2007) Economics on the Edge of Chaos, Environmental Modelling and Software, vol 22, pp. 580-589.

Gilmore C. G., (1993a), A new test for chaos, Journal of Economic Behaviour Organisations, Vol. 22, pp. 209-237.

Gilmore C. G., (1993b), A new approach to testing for chaos, with applications in finance and economics, International Journal of Bifurcation Chaos, vol. 3, n. 3, pp. 583-587.

Gilmore C. G., (1996), Detecting Linear and Nonlinear Dependence in Stock Returns: New Methods Derived From Chaos Theory, Journal of Business \& Accounting, vol. 29, (9) \& (10), pp. 1357-1377.

Gilmore C. G., (2001) An examination of nonlinear dependence in exange rates, using recent methods from chaos theory, Global Finance Journal, 12, pp. 139-151

Grandmont J. M., (1985), On endogenous competitive business cycles, Econometrica, vol. 50, pp.1345-1370.

Granger C. W. J., Terasvirta T., (1992) Experiments in Modeling Nonlinear Relationships Between Time Series, in Martin Casdagli and Stephen Eubank, eds., Nonlinear Modeling and Forecasting, Diagnostic Testing for Nonlinearity, Chaos, and General Dependence in Time Series Data. Redwood City, California: Addison-Wesley, 189-198.

Granger Clive W.J., (2001) Overview of Nonlinear Macroeconometric Empirical Models, Grassberger P., Procaccia I., (1983), Measuring the strangeness of strange attractors, Physica D, vol. 9, pp. 189-208.

Hentschel H. G. E, Procaccia I., (1983), The infinite number of generalized dimensions of fractals and strange attractors, Physica D8 pp. 435-444. 
Hinich M., (1982), Testing for Gaussianity and Linearity of a Stationary Time Series, Journal of Time Series Analysis, vol.3, iss. 3, pp.169-176.

Holyst A., Zebrowska M., (2000), Recurrence plots and Hurst exponents for financial markets and foreign exchange data, Internal Journal Theoretical Applied Finance, 3 pp. 419

Holyst A., Zebrowska M., Urbanowicz K., (2001), Observations of deterministic chaos in financial time series by recurrence plots, can one control chaotic economy, The European Physical Journal B 20, pp. 531-535

Hsieh D., (1989), Testing for non-linearity in daily foreign-exchange rate changes, Journal of Business, vol. 62, pp. 339-368

Hsieh D., (1991), Chaos and Nonlinear Dynamics: Applications to Financial Markets, Journal of Finance, vol. 46, iss.5, pp. 1839-1877.

Hsieh, D., (1993), Using Non-Linear Models to Search for Risk Premia in Currency Futures, Journal of International Economics 35, 113-132.

Iwanski J. S., E. Bradley (1998), Recurrence plots of experimental data: To embed or not to embed?, Chaos, vol. 8, pp. 861-871, http://www.cs.colorado. edu/users/lizb/papers/iwanskichaos98.pdf.

Jensen J. L., (1993), Chaotic dynamical systems with a view towards statistics: a review, in O.E. Barndor -Nielsen, J.L. Jensen, W.S. Kendall (eds.) Networks and Chaos, Statistical and Probabilistic Aspects, Chapman and Hall, London.

Kantz H., Schreiber T., (1997), Non linear time series analysis, Cambridge University Press.

Kellert S. H., (1992), A Philosophical Evaluation of the Chaos Theory Revolution, PSA:

Proceedings of the Biennial Meeting of the Philosophy of Science Association, vol. 2: Symposia and Invited Papers, pp. 33-49.

Kohzadi N., Boyd M.K., (1995), Testing for Chaos and Nonlinear Dynamics in Cattle Prices, Canadian Journal of Agricultural Economics, vol. 43, pp. 475-484.

Kuan G.M., (2008) Lecture on time series diagnostic tests, Institute of Economics Academia Sinica, February 17.

Kugiumtzis D (1997) Assessing different norms in nonlinear analysis of noisy time series. Physica D, vol. 105, pp.62-78.

Kyrtsou C., Labys W. C., M. Terraza, (2001) Noisy Chaotic Dynamics in Commodity Markets, Division of Resource Management Working Paper RESMWP-03-01.

Liu L., (2009)Testing for Nonlinearity and Chaoticity in Exchange Rate Time Series, SEI online.

Manetti C., Ceruso M., Giuliani a., Webber J. C., Zbilut J. P., (1999), Recurrence Quantification analysis as a tool for characterization of molecular dynamics simulations, Physical Review E 59, n.1.

Matilla-Garcià M., Queral R., Sanz P. and F.J. V. Zquez (2004) A Generalized BDS Statistic, Computational Economics 24, pp. 277-300.

Mayer-Kress G., (1986) Dimension and entropies in chaotic systems, ed., Springer-Verlag Berlin

Mayfield, E. S., B. Mizrach, (1992) On Determining the Dimension of Real-Time StockPrice Data, Journal of Business \& Economic Statistics, 10(3), pp. 367-374. 
McKenzie M. D., (2001), Chaotic behavior in national stock market indices, Global Finance Journal, vol. 12, iss.1, pp. 35-53.

Medio A., (1979) Teoria Non-Lineare del Ciclo Economico, Il Mulino, Bologna.

Mindlin G. B., Gilmore R., (1992), Topological Analysis and Synthesis on Chaotic Time Series, Physica D, 58, pp. 229-242.

Mindlin, G. B., Xin-Jun Hou, Hernán G. Solari, R. Gilmore, and N. B. Tufillaro (1990) Classification of strange attractors by integers, Physical Review Letters, vol. 64, pp. 23502353.

Mishra S. K., (2011) Volatility in Indian Stock Markets, Available at SSRN: http://ssrn.com/abstract=1788306.

Mizrach B., (1996), Determining delay time for phase space reconstruction with application to the FFDm exchange rate, Journal of Economic Behaviour \& Organisations, vol. $30 \mathrm{pp}$. 369-381.

Montoro J. D., J. V. Paz, (1997) Detecting macroeconomic chaos, Innovation in Mathematics: Proceedings of the Second International Mathematica Symposium, pp. 353360.

Nychka D. W., McCaffrey, Daniel F., Stephen P. Ellner, A. Ronald Gallant, and (1992), Estimating the Lyapunov Exponent of a Chaotic System with Nonparametric Regression, Journal of the American Statistical Association, vol. 87, pp.682-695.

Packard N. H., Crutcfield J. P., Farmer J. D., Shaw R. S., (1980), Geometry from a time series, Physical Review Letters 45, pp. 712-716.

Panas E., (2002), Long memory and chaotic models of prices on the London Metal Exchange, Resources Policy, vol. 27, pp. 235-246.

Panas U E., Ninni V., (2000), Are oil markets chaotic? A non-linear dynamic analysis, Energy Economics, vol. 22, pp.549-568.

Ramsey J. B., H. J. Yuan, (1989) Bias and error bars in dimension calculations and their evaluation in some simple models, Physics Letters A134, 397-398.

Ramsey J. B., H. J. Yuan, (1990) The statistical properties of dimension calculations using small data sets, Nonlinearity 3, 155-176.

Rosenstein M. T., Collins J. J., De Luca C. J., (1993), Reconstruction expansion as a geometry-based framework for choosing proper delay times, Physica D 65, pp. 117-134.

Savit R., Grenn M., (1991) Time series and dependent variables, Physica D, 50, pp. 95-116.

Sayers C. L., (1986), Work Stoppage: Exploring the Nonlinear Dynamics, Department of Economics, University of Houston.

Scheinkman J., B. LeBaron, (1989) Nonlinear Dynamics and Stock Returns, Journal of Business, University of Chicago Press, vol. 62(3), pp. 311-37.

Schwartz B. and S. Yousefi (2003) On Complex Behavior and Exchange Rate Dynamics, Chaos Solutions and Fractals, 18, p. 503-523.

Serletis A., Gogas P., (1997), Chaos in East European black-market exchange rates, Research in Economics, vol. 51, pp. 359-385.

Serletis A., Shintani M., (2003) No Evidence of Chaos but Some Evidence of Dependence in the U.S. Stock Market, Chaos, Solitons \& Fractals, 17, pp. 449-454. 
Serletis A., Shintani M., (2006), Chaotic monetary dynamics with confidence, Journal of Macroeconomics, 28, pp. 228-252

Sewell S.P., et al., (1996). Using Chaos Measures to Examine International Capital Market Integration, Applied Financial Economics, 6(2), 91- 101.

Shintani M., O. Linton, (2004) Nonparametric neural network estimation of Lyapunov exponents and a direct test for chaos, Journal of Econometrics, 120, pp. 1-33.

Stone M. A., (1989), Chaos, Prediction and LaPlacean Determinism, American Philosophical Quarterly, 26 (April), p. 123-131.

Streips M. A., (1995), The Problem of the Persistent Hog Price Cycle: A Chaotic Solution, American Journal of Agricultural Economics, vol. 77, pp. 1397-1403.

Strozzi F., Zaldivar J. M., Zbilut J. P., (2002), Application of nonlinear time series analysis techniques to high-frequency currency exchange data, Physica A 312, pp. $520-538$.

Stutzer M. J., (1980) Chaotic dynamics and bifurcation in a macro model, Staff Report 55, Federal Reserve Bank of Minneapolis.

Takens F., (1981), Detecting Strange Attractors in Turbulence, in Rand D.A., Young L.S., (Eds.), Dynamical Systems and Turbulence, vol. 898, Lecture Notes in Mathematics, Springer, Berlin, 1981, pp. 366--381.

Torkamani M. A., Mahmoodzadeh S., Pourroostaei S., and Lucas C. (2007) Chaos Theory and Application in Foreign Exchange Rates vs. IRR (Iranian Rial), World Academy of Science, Engineering and Technology, 30, pp. 328-332.

Trulla L. L., A. Giuliani, J. P. Zbilut, C. L. Webber Jr., (1996), Recurrence quantification analysis of the logistic equation with transients, Physics Letters A 223, pp. 255-260.

Tufillaro, N.B., Solari, H.G. and Gilmore, R., (1990) Relative rotation rates: fingerprints for strange attractors, Physical Review A 41, pp. 5717-5720.

Vaidyanathan R., Krehbiel T., (1992), Does the S\&P 500 Futures Mispricing Series Exhibit Nonlinear Dependence across Time?, The Journal of Futures Markets, vol. 12, pp. 659-677.

Wei A., Leuthold R. M., (1998), Long Agricultural Futures Prices: ARCH, Long Memory, or Chaos processes?, OFOR Paper, n. 03 May.

Wolf A., Swift J. B., Swinney H. L., Vastano J. A., (1985), Determining Lyapunov exponents from time series, Physica D, vol. 16, pp. 285-317.

Wu K., Savit R., Brock W., (1993) Statistical tests for deterministic effects in broad band time series, Physica D, 69, pp. 172-188.

Yang S. R., Brorsen B. W., (1992), Nonlinear Dynamics of Daily Cash Prices, American Journal of Agricultural Economics, vol. 74, pp. 706-715.

Yang S. R., Brorsen B. W., (1993), Nonlinear Dynamics of Daily Futures Prices: Conditional Heteroschedasticity or Chaos?, The Journal of Futures Markets, vol.13, pp. 175191.

Zbilut J. P., Giuliani A., Webber C. L. Jr, (2000), Recurrence quantification analysis as an empirical test to distinguish relatively short deterministic versus random number series, Physics Letters A, 267, pp. 174-178.

Zbilut J.P., Webber C.L. Jr. (1998), Detecting deterministic signals in exceptionally noisy environments using cross-recurrence quantification, Physics Letters A 246, pp. 122-128. 
Zbilut J.P., Webber C.L. Jr., (1992), Embeddings and delays as derived from quantification of recurrence plots, Physics Letters A 171, pp. 199-203. 
DEPARTMENT OF ECONOMICS AND PUBLIC FINANCE "G. PRATO" UNIVERSITY OF TORINO

Corso Unione Sovietica 218 bis - 10134 Torino (ITALY)

Phone: +390116706128 - Fax: +390116706062

Web page: http://eco83.econ.unito.it/prato/ 